\title{
La CRISIS AMBIENTAL EN MEDELLín, UNA PERSPECTIVA deSDE LA CONTABILIDAD
}

\section{The environmental crisis in Medellín, a perspective from accounting}

\author{
Edward Eli Loboa Ortega ${ }^{1}$, Niyiret Sastoque ${ }^{2}$, Yeimer Mora ${ }^{3}$
}

Recibido: Junio 22 de 2016/Aceptado: Septiembre 12 de 2016

\begin{abstract}
RESUMEN
Durante el año 2016, se declaró alerta roja ambiental durante 40 días en Medellín, debido a las alarmantes cifras que arrojaba el SIATA, respecto a la calidad del aire del Valle de Aburrá; repitiéndose durante la misma temporada luego de varias alertas naranjas, haciendo evidente la crítica situación que enfrenta esta subregión en materia de planeación y administración ambiental. El presente artículo, reflexiona entorno a posibles factores que desde el punto de vista económico-administrativo podrían influir positivamente en la transformación de esta dinámica, al analizar el actual plan de desarrollo con relación al tema, teniendo como base contabilidad ambiental, que ha sido objeto de estudio de la ciencia contable y en la cual todavía falta mucho camino por recorrer, principalmente en el caso colombiano, pero que permiten comprender esta problemática desde un punto de vista necesario $y$ poco frecuente en este tema.
\end{abstract}

Palabras clave: Medellín, Salud, Plan de Desarrollo, Responsabilidad ambiental, Calidad del aire, Contabilidad ambiental.

\section{ABSTRACT}

During the year 2016, declared alert environmental red for 40 days in Medellin, due to the alarming figures that threw the SIATA, with respect to the quality of the air of the Valley of Aburrá; repeating itself during the same season after several warnings oranges, making evident the critical situation faced by the sub-region in the field of planning and environmental management. This article reflects environment possible factors which could positively influence the transformation of this dynamic, analyzing the current development plan in relation to the subject, based from the administrative point of view environmental accounting, which has been the subject of study of accounting science and in which still a long way to go, mainly in the Colombian case, but allowing to understand this issue from a necessary point of view and some frequent in This topic.

Keywords: Medellin, Health, Development plan, Environmental responsibility, Air quality, Environmental accounting.

Cómo referenciar este artículo: Loboa, E., Sastoque, N. \& Mora, Y. (2016). La crisis ambiental en Medellín, una perspectiva desde la contabilidad. Ad-Gnosis, 5(5), 91-106.

1. Magíster en Negocios, Universidad del Norte. Profesional en Finanzas y Negocios Internacionales. Especialista en Finanzas. edwlob@hotmail.com

2. Abogada. Especialista y Magíster en Derecho Ambiental y Urbanos Territorial, Universidad del Norte. niyiretsastoque@hotmail.com

3. Contador Público, Universidad del Atlántico. Especialista en Gestión y Control Tributario, Universidad del Magdalena. Maestrante en Ciencias de la Administración y las Organizaciones, Universidad Tecnológica de Bolívar. yamg2163@hotmail.com 
Introducción

"Puede que seas capaz de engañar a los votantes, pero no a la atmósfera”.

El mundo hoy se encuentra, ante el reto de lograr que los poderes públicos y privados sean puestos al servicio del bienestar de los pueblos, de tal modo, que sea posible encontrar soluciones integrales para problemas que cada día se posicionan como temas de interés colectivo. Uno de estos es la exigencia de una responsabilidad social en materia ambiental, puesto que el tema de la sostenibilidad humana preocupa con razones de convenio, a diferentes sectores de la sociedad.

Ejemplo de ello es que durante mucho tiempo los recursos naturales fueron concebidos como un pozo sin fondo del cual podía nutrirse el desarrollo económico de las naciones; pero con el paso de los años se fue identificando el impacto ecosistémico y social que ello generó; por lo cual se hizo evidente que al no haber realizado un seguimiento cuidadoso a la manera en que se gastaron recursos renovables y no renovables que eventualmente comenzaron a escasear y desaparecer, se fue desequilibrando la balanza de la sostenibilidad humana sin saber qué era necesario hacer para encontrar de nuevo el punto de equilibrio.

\section{La contabilidad ambiental:}

"Todo lo que le ocurra a la tierra, le ocurrirá a los hijos de la tierra".

Muchas son las acciones que desde diversos sectores se han emprendido para aportar soluciones a esta problemática del mundo actual y en este orden de ideas se ha logrado reconocer que el Capital Natural, hace parte del Capital Total. Hoy es claro que al incluir este factor en el control de los procesos de producción no solo se logra identificar la relación anteriormente mencionada, sino que además se tiene mayor exactitud contable y financiera que permite conocer los impactos que se generan sobre los recursos naturales, e igualmente es posible identificar presupuestos necesarios para mitigar dichos impactos desde los sectores públicos y privados.

La existencia de numerosas leyes, decretos y resoluciones que pretenden subsanar las problemáticas ambientales; Colombia representa un marco de referencia con relación a los temas medioambientales, para otros países de Latinoamérica y el mundo. El Plan General de Contabilidad Pública, adoptado mediante la Resolución 355 de 2007, es muestra de ello, pues contiene las pautas conceptuales que deben observarse para la construcción del Sistema Nacional de Contabilidad Pública (SNCP); y en el cual se incluyen las normas técnicas en materia, financiera, económica, social y ambiental, reflejando de manera independiente los recursos aplicados en cada sector para las entidades públicas.

El propósito es proponer una reflexión, fundamentada sobre la manera en que se está articulando la gestión contable ambiental, al problema de sostenibilidad en materia ambiental 
que vive la ciudad de Medellín y que afecta directamente la salud pública, en relación con la problemática del aire; y de paso identificar posibles acciones complementarias que desde la normatividad contable podrían contribuir a mitigar el daño ambiental y prevenir el consecuente gasto público que generan las tasas actuales de enfermedad y muerte de las personas por afecciones relacionadas con la calidad del aire que se respira en la capital antioqueña y su área metropolitana.

Se puede realizar una pregunta clave de este tema: ¿Qué relación hay entre los ajustes al presupuesto destinado para la salud en el municipio de Medellín y los factores económicos nacionales que permitan justificar que dicho incremento no se corresponda con lo que se gasta ante las crisis ambientales y de salud pública de Medellín?, ayuda a conocer la realidad que se puede presentar durante este año y los años venideros por la súper-población automotora o ciudadana. Con el énfasis de que el medioambiente no se puede descuidar y no se puede dejar una calidad de vida sino se trabaja durante estos periodos que ayuden a mitigar este efecto. Así se puede mirar lo siguiente:

En su Plan de Desarrollo 2016 a 2019, MedeIlín destinó para la Salud durante el año 2016, la cifra global de $\$ 471.511 .663 .679$ de pesos, de acuerdo a lo registrado en el Proyecto Plan Operativo Anual de Inversiones -POAl- de este año (DAP Alcaldía de Medelín (A), 2016, p.4); y para el año 2017 fue aprobado un presupuesto de $\$ 506.650 .274 .669$ de pesos, según lo publicado en el sitio oficial de la Alcaldía de Medellín (Alcaldía de Medellin, 2016). Se puede observar para este año un incremento de $\$ 35.138 .610 .990$, un 6,89 \% con relación a la inversión del año anterior, un porcentaje ligeramente inferior al $7 \%$ que subió el Salario Mínimo Legal, lo cual representa una disminución presupuestal aparentemente mínima pero aun así preocupante, si se tiene en cuenta el aumento del costo de vida para el mismo año, junto al aumento de los impuestos, o la inflación, u otros factores externos como la salud de los habitantes, la crisis ambiental de la ciudad, así como las tasas de natalidad, morbilidad y mortalidad de este municipio de los años anteriores y la crisis nacional del sistema de seguridad social y salud, que afecta todos los aspectos administrativos que impactan el acceso de los colombianos a este derecho.

En el POAl del 2015, por su parte se evidencia una asignación presupuestal de \$456.233.522.350 (DAP Alcaldía de Medellín (B), 2015, p.3); mientras que en el de 2014 se puede observar que se asignó una cifra de \$399.331.000.000 (DAP Alcaldía de Medellín, 2015, p.105) y en el 2013 reposa una cifra de \$301.831.847.700 (DAP Alcaldía de Medellín, 2013, p.1).

Con esta información se puede establecer que en los tres años consecutivamente anteriores al periodo que se desea analizar en el presente texto (Municipio de Medellín, 2012, p.4; Municipio de Medellín, 2015, p.4; Municipio de Medellín, 2014, p.4) hubo un incremento de inversión en el sector salud de la siguiente manera: 
Tabla 1. La inversión en salud del 2013 al 2017en Medellín

\begin{tabular}{|c|c|c|c|}
\hline Año & Presupuesto Salud & Incremento anual en salud con relación año anterior & Valor del Incremento \\
\hline 2013 & 301.831 .847 .700 & Sin dato & Sin dato \\
\hline 2014 & 399.331 .000 .000 & $32,00 \%$ & $\$ 97.499 .152 .300$ \\
\hline 2015 & 456.233 .522 .350 & $12,00 \%$ & $\$ 56.902 .522 .350$ \\
\hline 2016 & 471.511 .663 .679 & $3,00 \%$ & $\$ 15.278 .141 .329$ \\
\hline 2017 & 506.650 .274 .669 & $6,94 \%$ & $\$ 35.138 .610 .990$ \\
\hline
\end{tabular}

Fuente: Elaboración propia a partir de datos tomados de los POAI 2013 al 2017

Según lo anterior se pueden mirar varios factores económicos que influyen en el presupuesto para la salud. Para darle contexto a lo que realmente significa e implica dicho incremento, cabe mencionar lo que aclaró la Ministra de Trabajo 2016, Clara López Obregón, cuando indicó que:

(...el incremento del $7 \%$ del Salario Mínimo Legal para el 2017 en Colombia...) Se trata de un incremento en términos reales de $1,5 \%$ frente a la proyección de la inflación del presente año, 5,5 \%, y seguramente de más de 2,5\% frente a las perspectivas para 2017, que sitúan la inflación entre el 4 \% y 4,5\%. (Ministerio del Trabajo, 2016)

Con la información consolidada para los incrementos en materia de salud en Medellín, se puede concluir de manera preliminar que no existe una coherencia entre los incrementos que se destinan a dicho sector y el aumento de la inflación o el costo de vida, lo cual no tendría mayor consecuencia si al menos se correspondiera con el estado de la salud entre los ciudadanos; pero en este texto se intenta- rá exponer que de hecho el panorama está al revés.

Para darle mayor peso a este argumento, resulta pertinente observar la Tabla 2, de elaboración propia a partir de la información pública sobre el valor establecido para los Salarios Mínimos Legales Vigentes (SMLV), año tras año; donde se ve la variación del SMLV durante los últimos cuatro años, según la cual se concluye que en los años de menor inversión de salud en la alcaldía de Medellín, el aumento del SMLV ha estado ubicado por encima del incremento a dicho sector, contrario a años anteriores (ver Tablas 1 y 2 ).

Tabla 2. Salario mínimo e Inflación en Colombia 2013-2017

\begin{tabular}{|c|c|c|c|}
\hline Año & $\begin{array}{c}\text { Salario mínimo } \\
\text { mensual }\end{array}$ & $\begin{array}{c}\text { Variación del } \\
\text { salario mínimo }\end{array}$ & $\begin{array}{c}\text { Inflación } \\
\text { del año }\end{array}$ \\
\hline 2013 & $\$ 589.500,0$ & $4,02 \%$ & $1,94 \%$ \\
\hline 2014 & $\$ 616.000,0$ & $4,50 \%$ & $3,66 \%$ \\
\hline 2015 & $\$ 644.350,0$ & $4,60 \%$ & $6,77 \%$ \\
\hline 2016 & $\$ 689.455,0$ & $7,00 \%$ & $5,75 \%$ \\
\hline 2017 & $\$ 737.717,0$ & $7,00 \%$ & $\begin{array}{c}\text { No se genera } \\
\text { dato aún }\end{array}$ \\
\hline
\end{tabular}

Fuente: Elaboración Propia. Datos abiertos de la Presidencia de la República, disponibles para consulta pública en los sitios web oficiales del Banco de la República y el Ministerio de Trabajo.

(Banco de la República - Colombia, 2013) (Ministerio del Trabajo - Colombia, 2013) 
La situación de salud en el contexto local y regional 2014-2017

Llama mucho la atención dicha inversión y sus variaciones en los últimos cuatro años, si se tiene en cuenta lo que reza el Plan de Desarrollo Vigente del municipio, en cuanto a la situación de salud de la ciudad, pues se puede observar de entrada que se reconoce que existen múltiples fenómenos que le afectan e implican una mayor gestión de recursos alrededor suyo:

Uno de los indicadores tradicionales para medir el estado de salud de una población (...) son los Años de Vida Potencialmente Perdidos por causa de enfermedad evitable (AVPP), que para 2014 llega a los 9,6 años por cada 100.000 habitantes. Sus principales causas fueron el tumor de mama, seguido de las enfermedades crónicas de las vías respiratorias inferiores y las infecciones respiratorias agudas. En este caso los causales fueron las enfermedades no transmisibles y las lesiones generales. (DAP Alcaldía de Medellín (A), 2016, p222)

La anterior información es complementada en dicho texto con la siguiente ilustración que se extrae de allí de manera directa, para mayor claridad frente a dichas cifras:

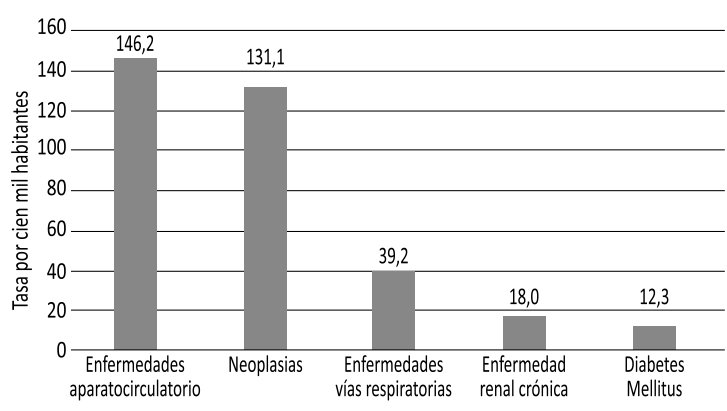

Figura 1. Principales causas de mortalidad en Medellín desde el 2014

Fuente: Imagen extraída del documento Medellín como Vamos (DAP Alcaldía de Medellín (A), 2016, p.222)

Es decir, que para la administración es claro que se está observando que las enfermedades de las vías respiratorias (no transmisibles) consideradas de carácter crónico y agudo, es decir, aquellas que requieren una intervención médica prioritaria tienen un papel protagónico en las cifras de mortalidad de Medellín.

Es importante aquí aclarar que estas son tipificadas y reportadas por entidades prestadoras de salud obligatoriamente en el SIVIGILA, que es un sistema que se ha creado para realizar la provisión en forma sistemática y oportuna, de información sobre la dinámica de los eventos que afecten o puedan afectar la salud de la población colombiana; por lo cual resulta ser una fuente confiable para establecer las cifras y los datos en torno a salud pública.

De este sistema (SIVIGILA, 2015-2017) se han extraído algunos reportes que se consignan a continuación con el interés de mostrar de la manera más objetiva posible, de qué se trata la crisis de salud relacionada con factores ambientales que se menciona en el presente tex- 
to, y corroborar la información consignada en la fuente información citada anteriormente:

Tabla 3. Reporte del SIVIGILA diagnósticos y muertes asociadas a IRA, ESI e IRAG entre 2015 y 2016

\begin{tabular}{|c|c|c|c|}
\hline Reporte del SIVIGILA & Antioquia & Medellín & AMVA \\
\hline \multicolumn{4}{|c|}{ Casos reportados durante el 2015} \\
\hline Casos ESI - IRAG (V. C) & 686 & 480 & 540 \\
\hline $\begin{array}{l}\text { Infección Respiratoria } \\
\text { Aguda Grave IRAG } \\
\text { Inusitada }\end{array}$ & 366 & 164 & 254 \\
\hline Mortalidad por IRA & 1123 & 23 & 36 \\
\hline \multicolumn{4}{|c|}{ Casos reportados durante el 2016} \\
\hline Casos ESI - IRAG (V. C) & 404 & 306 & 344 \\
\hline $\begin{array}{l}\text { Infección Respiratoria } \\
\text { Aguda Grave IRAG } \\
\text { Inusitada }\end{array}$ & 167 & 69 & 96 \\
\hline Mortalidad por IRA & 53 & 15 & 19 \\
\hline
\end{tabular}

Fuente: Elaboración propia a partir de datos disponibles en SIVIGILA (SIVIGILA, 2015-2016)

Para interpretar la Tabla 3, es pertinente informar que IRA es Infección Respiratoria Aguda y ESI - IRAG es Enfermedad Similar a la Influenza - Infección Respiratoria Aguda Grave; y ambas pueden ocasionar la muerte. Igualmente es importante mencionar que no se incluyen en el SIVIGILA las cifras de las enfermedades que se mezclan con cuadros virales o bacterianos, ni aquellas que no llegan a los centros hospitalarios y por ende no son tenidas en cuenta a la hora de consolidar datos con respecto a la salud respiratoria de los medellinenses; por lo cual es posible concluir que estas cifras son inferiores a las reales, como sucede por lo general en cuanto a datos estadísticos de salud pública en Colombia, debido a las barreras culturales y económicas, de acceso a la salud para ciertos grupos poblacionales.
Tampoco se incluyen en este reporte, quienes viven en municipios cercanos que no hacen parte del AMVA pero que igualmente habitan Medellín, como lo son las personas de Guarne, Rionegro, Marinilla, El Retiro, y La Ceja que estudian y/o trabajan en el AMVA pero que son tenidos en cuenta en las cifras de los municipios a los que pertenece la IPS a la que acuden por temas de salud, y no en esta subregión.

Así pues, que no se debe perder de vista que los datos oficiales son inferiores a los datos reales, que no se incluyen porque no se pueden tipificar dados los cruces de datos azarosos, pero es necesario indicar aquí que, de hacerlo, se prendería una alarma roja, en una situación que de por sí ya resulta inquietante, puesto que la cantidad de muertes por enfermedades respiratorias asociadas a otros diagnósticos es realmente muy alta.

\section{Factores asociados a la crisis del aire que vive el AMVA y Medellín como epicentro}

De acuerdo con el Área Metropolitana del Valle de Aburrá, organización administrativa que regula el desarrollo urbano y ambiental de los 10 municipios del Área Metropolitana, Medellín se encuentra en la mitad del Valle, en medio de las montañas que componen la Cordillera Central de la porción colombiana de Los Andes. Un valle estrecho, atravesado de norte a sur por el río Medellín, en cuyo centro se localiza el área urbana de Medellín, abarcando las zonas bajas e intermedias de las montañas, y alrededor de las montañas (Área Metropolitana del Valle de Aburrá, 2016). 
El poblamiento de esta subregión antioqueña se dio gradualmente desde el Norte y desde el Sur hacia el centro del Valle de Aburrá, donde se construyeron los primeros poblados a la par que se instauraron las primeras fábricas de una de las ciudades más industrializadas del país. Por lo anterior, no resulta extraño encontrar en el centro de la ciudad y en las zonas que rodean al río Medellín, la mayor densidad de fábricas humeantes que desde hace décadas trabajan con chimeneas medianas y pequeñas, incluso por debajo de la altura legal permitida en otras ciudades del mundo y por la normatividad actual del país.

Con una topografía bastante escarpada en sus costados, la ciudad no cuenta con vías circunvalares ni un sistema vial periférico que conecte sus alrededores, tal y como sucede en muchas ciudades igualmente industrializadas, y de hecho, debido a estar atravesada por un gran río, las calles que se construyen responden más a los caprichos de su naturaleza que a los intereses de una planeación ordenada del territorio, ante lo cual la ciudad experimenta constantes dificultades de movilidad que invitan a los habitantes a querer desplazarse en vehículos propios, lo cual genera un incremento gradual y descontrolado del parque automotor, y los gases que estos arrojan al aire diariamente.

Otro aspecto relevante de la ciudad y su naturaleza, es el hecho de que los vientos que la atraviesan normalmente van de norte a sur, mientras que sus aguas van de sur a norte, por lo cual resulta complicado que se haga un barrido natural eficiente de los gases que se acumulan en el centro del valle, y cuya salida se hace compleja debido a las grandes montañas que se constituyen en barreras naturales al oriente y al occidente del mismo.

Por otra parte, Medellín es una ciudad densamente poblada, la tercera del Rankin Mundial, de acuerdo con el último informe del Foro Económico Mundial (WEF - World Economic Forum, 2016), lo que se nota en los costos del metro cuadrado con relación al sector donde se piense construir, algo que permite comprender la escasez de suelos no construidos (lotes limpios) en las partes bajas del valle y el creciente reemplazo de zonas verdes por urbanizaciones y edificios, en los barrios tradicionales, e incluso en zonas que durante décadas representaron pulmones para la ciudad pero que hoy están siendo eliminadas para dar paso a proyectos de vivienda horizontal.

Es interesante mencionar aquí, que la información del WEF consultada, explica en gran parte que su zona urbana no es ambientalmente sostenible y que el hecho de estar rodeada de otros municipios que crecen al mismo ritmo, genera una serie de desequilibrios entre el aire que se ensucia y el que logra escapar del caldero de vapores, gases y demás residuos que quedan suspendidos en el aire o que se cuecen en el interior de este valle de fábricas y concreto.

Residuos que si no encuentran vientos resis- 
tentes y las condiciones climáticas adecuadas en cuanto a temperatura y densidad de las nubes que se posan sobre el valle de tal modo que logren empujar el smog afuera del valle; se harán cada vez más densos al unirse con otras partículas de residuos que se liberan diariamente.

Es decir, que Medellín no cuenta actualmente con unas condiciones geográficas, demográficas, de movilidad, ni ambientales, que les permitan a sus habitantes respirar un aire limpio o con condiciones aceptables para garantizar una buena salud en sus ciudadanos. De hecho, para garantizar un aire menos contaminado en Medellín, es necesario que haya un clima intermedio-templado, con vientos fuertes, puesto que cuando sube mucho la temperatura los vientos se disipan y las partículas se quedan suspendidas en el aire, y cuando desciende la temperatura, las nubes funcionan como un techo que encierra los gases y vapores, y agrava igualmente la situación de toxicidad del aire, en cuanto incrementa la cantidad de partículas dañinas en el aire.

Dado que la temperatura en la ciudad se presenta muy inestable debido a fenómenos de la región, tales como el niño y la niña, y además debido a la polución que se concentra bajo las nubes de agua, enfriando las partículas de residuos, uniéndolas, densificándolas y saturando el aire a niveles que el cuerpo no puede procesar, junto con otros episodios que sobrecalientan el clima, van generando cuadros de infecciones respiratorias graves y crónicos en los pobladores, que terminan por incrementar la morbilidad y mortalidad.

De modo que si no se tienen siempre en cuenta todos los anteriormente enunciados, sino que se orientan las acciones de mitigación del impacto hacia solo uno o dos de los factores, será imposible generar un plan de acción efectivo y esto lo saben las autoridades ambientales.

\section{Soluciones implementadas y tareas pendien-} tes con relación al aire de Medellín

El panorama es grave, pero resulta aún más preocupante si se tiene en cuenta que las mitigaciones ambientales que se proponen y realizan, están orientadas a acciones como la siembra masiva de árboles, lo cual está bien en cuanto un árbol adulto refresca, mejora el curso de los vientos, regula las temperaturas y limpia gran parte del aire del $\mathrm{CO} 2$, pero no resuelve el problema más grave, ya que por un lado muy pocos árboles adultos quedan en esta ciudad, y por otro lado, porque no existen árboles capaces de transformar en oxígeno el material particulado (PM) que se encuentra suspendido en el aire y que básicamente se compone de residuos de metal o caucho, y que ponen a la ciudadanía en alto riesgo de sufrir de enfermedades respiratorias, cardiopatías y accidentes cerebro vasculares.

En relación con este último punto, del material articulado concentrado en el aire que se respira al interior del Valle del Aburrá, la Organización Mundial de la Salud (OMS) recomien- 
da utilizar como indicador de la concentración de partículas materiales en el aire los valores de las partículas de diámetro inferior a 2,5 micras, PM 2,5, frente a los valores utilizados hasta ahora de las partículas de diámetro inferior a 10 micras, PM 10.

Linares y Díaz (2008) indican que el material particulado respirable presente en la atmósfera de las ciudades en forma sólida o líquida (polvo, cenizas, hollín, partículas metálicas, cemento y polen, entre otras) se puede dividir, según su tamaño, en dos grupos principales. A las de diámetro aerodinámico igual o inferior a los $10 \mu \mathrm{m}$ o 10 micrómetros ( 1 um corresponde a la milésima parte de un milímetro) se las denomina PM 10 y a la fracción respirable más pequeña, PM 2,5. Estas últimas están constituidas por aquellas partículas de diámetro aerodinámico inferior o igual a los 2,5 micrómetros, es decir, son 100 veces más delgadas que un cabello humano (Linares \& Díaz, 2008). Esta información resulta pertinente porque existe una tendencia muy fuerte en la ciudadanía, a creer que sembrar árboles es la cura para todos los males. La página web del Parque Explora, el Museo Interactivo de Ciencia de Medellín, presenta la siguiente información de manera muy clara, para comprender mejor la relación salud/PM:

Por un lado, las partículas de más de 10 $\mu \mathrm{m}$ de diámetro, (...) son filtradas por los vellos de la nariz y las mucosas del organismo. Por otro lado, aquellas con un diámetro menor -bacterias, humo de tabaco, virus, hollín, entre otros- tienden a permanecer suspendidas por un tiempo mayor: de minutos a horas en el caso de las que tienen de 2,5 a $10 \mu \mathrm{m}$ de diámetro (PM 10), y de horas a semanas en el caso de las menores de 2,5 $\mu \mathrm{m}$ (PM 2,5). (Vásquez-Muriel, 2016)

Explica que dichas partículas pueden entrar al cuerpo más fácilmente y pueden permanecer en los pulmones por mucho tiempo o pasar al torrente sanguíneo y distribuirse por todo el cuerpo, generando disfunciones a corto, mediano y largo plazo. Los principales efectos asociados a la exposición al PM 10 y, sobre todo, PM 2,5 incluyen: mortalidad prematura, enfermedades cardiovasculares, asma agravada, bronquitis crónica, función pulmonar disminuida y mayor riesgo de infarto cardíaco (Vásquez-Muriel, 2016).

$Y$ finalmente, se indica en el mismo sitio, que el aire de Medellín en los últimos años ha presentado niveles alarmantes de PM 10 y 2,5, debido a múltiples factores, y que las principales corrientes de aire de la ciudad no son suficientes para sacar estas partículas. Finaliza indicando que como el aire no circula ni se limpia, reducir las emisiones de la ciudad es la única manera de contrarrestar la contaminación (Vásquez-Muriel, 2016).

Ahora bien, con respecto a la relación entre este material articulado y la salud de los habitantes que respiran el aire impregnado de esto, un estudio de la Universidad de Antioquia (Ga- 
viria, Benavides \& Tangarife, 2011) demuestra evidencias estadísticas de la relación positiva entre contaminación por material articulado PM 2,5 y PM 10 y las consultas externas y por urgencia debidas a enfermedades respiratorias (asma, bronquitis, infecciones, rinitis). Confirmando así que las cifras alarmantes del SIVIGILA que se expusieron anteriormente sí guardan una estrecha relación con la calidad del aire. De hecho, concluye señalando que resulta lamentable que no existan estudios que permitan valorar el impacto sobre los sistemas de salud que acarrean los problemas ambientales ni los problemas económicos que se generan con cada crisis ambiental, dejando que además tampoco existe una forma de verificar los daños a largo plazo que el deterioro del aire genera en la salud y la economía de la ciudad:

El problema de la contaminación, dados sus efectos en la salud humana, se considera un problema de salud pública con consecuencias significativas sobre la tasa de morbilidad y mortalidad (por causas no violentas) en la ciudad. Así, este tipo de estudio puede replicarse para otros contaminantes relacionados con emisiones móviles y su incidencia en la salud humana, considerando que probablemente los efectos de la exposición no se manifiestan de forma inmediata; ello puede tardar varios días. (Gaviria, Benavides \& Tangarife, 2011, p.249)
Se trata de un estudio publicado en el 2011, es decir, en una época previa a las ya acostumbradas crisis ambientales; lo cual deja claro que en efecto existen desde hace varios años indicios que permitirían hacer una mejor planeación en cuanto a la relación entre lo que necesitaría hacer la ciudad en función de las medidas ambientales de modo que la salud de los habitantes no se vea afectada como hasta ahora ha sucedido, y de esta manera reducir los costos que se han generado tanto por la atención a los pacientes así como otros gastos asociados con el tema ambiental.

Del mismo modo, un estudio de la Universidad de Medellín del 2008, realizó un análisis similar y a partir de prácticamente los mismos hallazgos terminó recomendando como necesarias las siguientes medidas frente a este fenómeno:

Incorporar las mediciones de partículas PM 2,5 a la gestión ambiental e incluirla en estudios epidemiológicos, dado que es la fracción más tóxica de las partículas totales, porque provienen de los procesos de combustión y, además, por el tamaño de las partículas, que son capaces de penetrar hasta los alvéolos pulmonares $y$, por ende, tienen mayor incidencia en la salud. A corto plazo la Red de Vigilancia de la Calidad del Aire (REDAIRE) debe adecuarse para realizar el seguimiento de partículas finas (PM 2,5) en Medellín. (Echeverri-Londoño \& Maya-Vasco, 2008, p.41) 
Estas recomendaciones, que además son parte de una investigación rigurosa, fueron dadas hace casi 10 años, a partir del análisis tanto de los datos de dicho estudio, como de recomendaciones de otros estudios similares; pero hoy no están aplicándose ni la mitad de estas.

Pareciera que se sigue respondiendo solo de manera reactiva, es decir, como respuesta a la alarma, y no de manera proyectiva, lo cual, desde el punto de vista de la gestión administrativa y contable, genera pérdidas económicas para los sectores públicos y privados así como para la ciudadanía y ocasiona desaprovechamiento de los recursos así como gastos ineficientes del presupuesto público.

Ejemplo de esto es que durante la alerta ambiental del 2016 lo que se implementó como medida, y que ha sido la forma en que se viene mitigando el impacto ambiental, ha sido controlando el flujo vehicular durante las temporadas más frías y más cálidas del Valle de Aburrá (cerca de marzo y cerca de octubre y en mitad de año y final de año) imponiendo mayor control sobre los vehículos de dos y cuatro ruedas que se desplazan por el valle de Aburrá, y sancionando a quienes no tienen en regla las emisiones de gases de sus vehículos.

Sin embargo, esto no resulta del todo útil, puesto que existen cientos de chimeneas legales y otras muchas ilegales que operan sin filtros adecuados e impregnan el aire de PM que resulta altamente dañino para la salud de Medellín, y dichas medidas que se dan solo durante unos pocos días, al no ser continuas se convierten más en un pañito de agua tibia que en una solución real y a largo plazo.

Pero además, es necesario tener en cuenta que dichos paliativos, aunque sean mínimos generan mucho escozor entre los comerciantes, toda vez que les impone ser flexibles con los horarios tanto de sus empleados como de sus empresas y bajar la productividad a la cual están acostumbrados, en función de darle un respiro al aire, lo que para ellos se traduce en pérdidas.

Por ejemplo, Sergio Ignacio Soto, director de la Federación Nacional de Comerciantes, FENALCO citado por El Colombiano, se pronunció respecto a las medidas, argumentando que estas afectan más al comercio y por ende al bolsillo de lo que realmente le aportan a mejorar la calidad del aire y por ende a la salud de los habitantes del Valle de Aburrá destacando que:

En los primeros tres días de pico y placa ambiental, según los comerciantes, la productividad multisectorial del Valle de Aburrá dejó de obtener ingresos cercanos a $\$ 100.000$ millones, lo cual representa para ellos pérdidas por $\$ 20.000$ millones. Los cinco días adicionales de la medida que comienza hoy y va hasta el viernes 31 causan preocupación, aunque reconocen los beneficios ambientales. Por otro lado, es poco el tiempo para evaluar resultados económicos de- 
finitivos ya que incluso se tendrían que considerar otros factores como el clima. (Y) afirmó que la industria, los servicios y el comercio en el Valle de Aburrá mueven al día unos $\$ 100$ mil millones. Con el pico y placa, detalla, dejaron de tener ingresos (no son pérdidas) por \$33 mil millones o sea que en los tres días Soto calculó la cifra en $\$ 100$ mil millones. Un $20 \%$ de este valor, sostuvo el dirigente, sí fueron de pérdidas que suman $\$ 20$ mil millones. (Martínez Arango, 2017)

En este sentido, se observa un punto de quiebre llamativo entre los intereses económicos de la empresa privada y el bienestar de la ciudadanía, que de alguna manera también representan los intereses económicos de la ciudad, que permite comprender la importancia de este tema.

Otros de los esfuerzos se han concentrado en movilizar a las personas en el sistema Metro de manera gratuita, lo cual a simple vista parece una excelente solución, pero que generó un gasto importante en los recursos, puesto que el alcalde Federico Gutiérrez precisó, de acuerdo con el diario El Colombiano, en la misma columna donde se citaba al presidente de FENALCO, para hablar de este tema, que: Durante esos días - del 1 al 6 de abril-se calculaban 1'200.000 viajes y la cifra fue de 2' 400.000 viajes. Se calcula que el metro dejó de recibir \$1.700 millones, pero eso lo puede compensar el Área Metropolitana (Ospina Zapata, 2016).

\section{Reflexiones desde la contabilidad ambiental} de cara a la crisis del aire en Medellín

La Contabilidad Ambiental definida por Juan Antonio Lezca (2003), es una disciplina que se orienta hacia:

Mejorar el nivel de comunicación, las normas de registro y gerenciamiento contable, así como la contribución contable a las prácticas de administración del asunto ambiental en las empresas y descontaminar los balances que hoy omiten activos consumidos ambientales y que precisan ser mensurados y registrados. (Lezca, 2003)

La cual, del mismo modo en que sucede con la contabilidad tradicional, hace uso de herramientas como las cuentas ambientales, los informes financieros y gerenciales; para lograr el objetivo de medir los hechos económicos que afectan a la empresa y así proporcionar "información fidedigna, confiable, oportuna, comprensible, objetiva e integra" (Lezca, 2003). Por lo tanto, las cuentas ambientales resultan ser la mejor herramienta para que la contabilidad ambiental pueda vincularse a la gestión contable, y de este modo los temas ambientales sean factores de importancia financiera para las empresas públicas y privadas. Sin embargo, aún no se cuenta con una adecuada clasificación y registro de los diferentes conceptos ambientales.

La anterior información está referenciada por la tesis de grado Hacia la construcción de una 
contabilidad ambiental para Colombia, elaborada en 2009, por los contadores públicos Elkin Echeverry Villa, Astrid Suley Moná Ciro y Frank Rivillas Correa, egresados de la Universidad de Antioquia, quienes además complementan lo enunciado por Lezca argumentando que:

Los informes financieros en el contexto de la contabilidad ambiental darán cuenta de los activos, pasivos, patrimonio, ingresos, costos y gastos ambientales que se presenten en las empresas y de la cual todos los usuarios de la información contable deban tener conocimiento, a fin de facilitar la toma de decisiones. (Echeverry-Villa, Moná \& Rivillas-Correa, 2009, p.7)

En efecto, en Colombia se cuenta con el Plan General de Contabilidad Pública, adoptado mediante la Resolución 355 de 2007, que contiene las pautas conceptuales que deben observarse para la construcción del Sistema $\mathrm{Na-}$ cional de Contabilidad Pública (SNCP), y existe el Sistema de Contabilidad Ambiental y Económica, que incluye una Cuenta Satélite Ambiental (CSA) la cual de acuerdo a lo enunciado en el sitio web del DANE tiene como objetivo general:

Medir en unidades físicas y monetarias, de forma sistémica y para cada período contable, la variación de los stocks de los activos ambientales, las interacciones entre el ambiente y la economía, dentro de la economía y de la econo- mía al ambiente. (...) y mide el esfuerzo de los diferentes sectores económicos para conservar, mitigar o proteger el medioambiente. (DANE, 2016)

Pero hay que tener en cuenta que los desarrollos en el tema de cuentas ambientales en Latinoamérica no ha avanzado a pasos agigantados, y por ello no es frecuente observar cuentas satélites ambientales articuladas al sistema nacional de cuentas. $O$ se observan esfuerzos incompletos, como el caso colombiano. La CGN (Contaduría General de la Nación) planea y ejecuta un conjunto de actividades que prevengan la contaminación y contribuyan a proteger, conservar y fomentar la preservación del medioambiente. La política ambiental de la CGN se implementa a través del SGA (Sistema de Gestión Ambiental) y tiene como elementos básicos la definición periódica de objetivos y metas ambientales, el cumplimiento de la legislación ambiental y el compromiso de la Dirección de mejorar continuamente, pero ello aplica mayoritariamente para el sector público.

\section{Conclusiones}

- De modo que, si es obligación del Estado a garantizar un ambiente sano, no se está garantizando el derecho que le corresponde a la ciudadanía a disfrutar de un ambiente sano, y es por esta razón que se consideró pertinente indagar sobre qué tan comprometido está el AMVA y en específico el municipio de Medellín con la causa ambiental.

- Medellín ha tocado el límite ambiental, y si 
bien el valle de Aburrá no es la única región de Colombia con un aire poco apto para ser respirado, sí es la única que toma registro sistemático del material particulado suspendido en el aire; lo cual puede, este panorama llevar a declarar constantes alertas ambientales, que por supuesto generan molestia en la ciudadanía, pues de hecho es la única ciudad que ha declarado alertas rojas para que sus habitantes tomen medidas ante los problemas de la calidad del aire. Si bien es negativo para la ciudadanía tener que entender cuál es la realidad del aire que respira, resulta admirable que la ciudad asuma una posición tan abierta frente al tema.

- En materia de informar a los ciudadanos de manera oportuna y clara sobre el estado de la calidad de su aire es bueno para la ciudad, pero deja en evidencia que como ciudad responsable de proteger y cuidar la salud de sus habitantes, no se está haciendo la tarea.

- Ante los llamados de atención por parte del gremio de la salud así como de colectivos de ciudadanos que piden mayor responsabilidad ambiental, pero igualmente debido a las alertas de los tecnológicos sistemas de monitoreo implementados, la ciudad ha empezado a responder; pero no es suficiente el esfuerzo cuando se observa que los daños que genera la mala calidad del aire, tienden a ser cada vez mayores debido en parte a la topografía del valle en que se asienta el municipio y su área metropolitana.
- Teniendo en cuenta la política Ambiental de CGN, se puede deducir que aunque la entidad ya está implementando el uso de la contabilidad ambiental en Colombia, también es claro que todas las organizaciones ya hablan de contabilidad ambiental y están trabajando en su implementación interna para aportar al tema global, pero es tan poco lo que se ha avanzado en el tema y se ha convertido algo complejo si se tiene en cuenta la transición por la que están pasando las empresas con la implementación de las Normas Internacionales de Información Financiera.

\section{Referencias}

Alcaldía de Medellín (22 de 10 de 2016). Página Web de la Alcaldía de Medellín. Obtenido de https://www.medellin.gov. $\mathrm{co} / \mathrm{irj} /$ portal/medellin?NavigationTarget=navurl://7244969f35eda532b22e$5 \mathrm{fdfbad} 506 \mathrm{f} 8$

Alcaldía de Medellín (2016). Plan de Desarrollo Municipal 2016-2019: Medellín cuenta con vos. Alcaldía de Medellín, Antioquia. Medellín: Municipio de Medellín. Recuperado el 30 de 05 de 2016, de https://www.medellin.gov.co/irj/ go/km/docs/pccdesign/SubportaldelCiudadano_2/PlandeDesarrollo_0_17/ Publicaciones/Shared\%20Content/Documentos/2016/Proyecto\%20de $\% 20$ Acuerdo\%20Plan\%20de\%20Desarrollo. pdf

Área Metropolitana del Valle de Aburrá (20162019). Plan de Gestión. Recuperado de 
http://www.metropol.gov.co/institucional/planes/Plan\%20de\%20Gestion\%20 2016-2019.pdf

Banco de la República (2018). Salario Mínimo Legal en Colombia. Recuperado de: http://www.banrep.gov.co/es/mercado-laboral/salarios

DANE (2016 de 07 de 2016). Cuenta Satélite Ambiental. Obtenido de Departamento Nacional de Estadística: https:// www.dane.gov.co/index.php/estadisticas-por-tema/ambientales/cuenta-satelite-ambiental-csa

DAP Alcaldía de Medellín (2013). Plan Operativo Anual de Inversiones. Medellín: Alcaldía de Medellín. Recuperado el 25 de 05 de 2015, de http://www.concejodemedellin.gov.co/concejo/concejo/file_ downloader.php?id_file=18979-concejo-77c37ab61477be309f01501cbb6411fb

DAP Alcaldía de Medellín (B) (2015). Plan Operativo Anual de Inversiones. Departamento Administrativo de Planeación. Medellín: Alcaldía de Medellín. Recuperado el 29 de 05 de 2016, de https:// www.medellin.gov.co/irj/go/km/docs/ pccdesign/SubportaldelCiudadano_2/ PlandeDesarrollo_0_17/Publicaciones/Shared\%20Content/Documentos/2015/POAI\%202015.pdf

DAP Alcaldía de Medellín (A) (2016). Plan Operativo Anual de Inversiones. Departamento Administrativo de Planeación. Alcaldía de Medellín. Recuperado el 05 de 06 de 2016, de https://www.mede-
Ilin.gov.co/irj/go/km/docs/pccdesign/ SubportaldelCiudadano_2/PlandeDesarrollo_0_17/Publicaciones/Shared\%20Content/Documentos/2016/ POAI_2016_LCP.pdf

Echeverri Londoño, C. A. \& Maya Vasco, G. J. (2008). Relación entre las Partículas Finas (PM2.5) y Respirables (PM 10) en la diudad de Medellín. Redalyc.org. Recuperado de: http://www.redalyc.org/ html/750/75011517002/

Echeverry, E., Moná, A. \& Rivillas, F. (2009). Hacia la construcción de una contabilidad ambiental para Colombia: Un estudio comparativo a partir del caso Español (p.7). Universidad de Antioquia. Recuperado de: http://aprendeenlinea. udea.edu.co/revistas/index.php/tgcontaduria/article/view/323587/0

Foro Económico Mundial (2016). Foro Económico Mundial sobre América Latina. Recuperado de: https://www.weforum. org/es/agenda/2016/06/en-vivo-foro-economico-mundial-sobre-america-latina-2016/

Gaviria, C., Benavides, P. \& Tangarife, C. (2011). Contaminación por material particulado $\left(P M_{2,5}\right.$ y $\left.P M_{10}\right)$ y consultas por enfermedades respiratorias en Medellín (2008-2009). Scielo. Recuperado de: http://www.scielo.org. co/scielo.php?script=sci_arttext\&pi$d=$ S0120-386X2011000300004

Lezca, J. A. (2003). Contabilidad Ambiental. Apunte Contable. Revistas Universidad Externado de Colombia. Recuperado 
de: http://revistas.uexternado.edu. co/index.php/contad/article/viewFile/1299/1236

Linares, C. \& Díaz, J. (2008). Las PM $(2,5)$ y su afección a la salud. Recuperado de: https://www.um.es/estructura/servicios/ sprevencion/c-seguridad/documentos/ EE_58_PM25.pdf

Martínez Arango, R. (2017). Pico y placa afecta productividad. El Colombiano. Recuperado de: http://www.elcolombiano. com/antioquia/pico-y-placa-afecta-productividad-NC6217807

Ministerio del Trabajo (2013). Salario Mínimo Legal Vigente. Decretos. Recuperado de: http://www.mintrabajo.gov.co/documents/20147/36487/decreto_3068_ del_30_de_Diciembre_de_2013.pdf/358a653c-5346-f782-3f96-b044362fcee3

Ministerio del Trabajo (2016). Gobierno decreta aumento del $7 \%$ para salario mínimo. El Heraldo. Recuperado de: https:// www.elheraldo.co/colombia/gobierno-decreta-aumento-del-7-para-salario-minimo-315659
Municipio de Medellín (2012-2015). Plan de Salud Municipal. Recuperado de: https://www.medellin.gov.co/irj/go/km/ docs/pccdesign/SubportaldelCiudadano_2/PlandeDesarrollo_0_15/InformacinGeneral/Shared\%20Content/Documentos/instrumentos/ps/PLAN\%20 DE\%20SALUD\%20MUNICIPAL.pdf

Organización Mundial de la Salud (OMS) (2016). Calidad del Aire Ambiente (exterior y salud). Recuperado de: http:// www.who.int/mediacentre/factsheets/ fs313/es/

Ospina Zapata, G. (2016). Sigue pelea entre Alcalde y Gobernador por el metro gratis. El Colombiano. Recuperado de: http://www.elcolombiano.com/antioquia/movilidad/sigue-pelea-entre-alcalde-y-gobernador-por-el-metro-gratis-DX4077603

Vásquez Muriel, D. (2016). Nuestra Sangre como el Aire de Medellín. Parque Explora. Recuperado de: http://www.parqueexplora.org/aprende/actualidad/nuestra-sangre-como-el-aire-de-medell-n 